\title{
Comment on the original article entitled "Ethanol lock is effective on reducing the incidence of tunneled catheter-related bloodstream infections in hemodialysis patients: a systematic review and meta- analysis"
}

\author{
Peng Zhang ${ }^{1} \cdot$ Xin-Jun Su$^{1} \cdot$ Xing-Huan Wang $^{1}$
}

Received: 23 April 2018 / Accepted: 10 May 2018 / Published online: 7 June 2018

(c) Springer Science+Business Media B.V., part of Springer Nature 2018

\section{Editor,}

We recently read a paper that was published in your journal and entitled "ethanol lock is effective on reducing the incidence of tunneled catheter-related bloodstream infections in hemodialysis patients: a systematic review and meta-analysis" by Zhao et al. [1], and we found some issues that need to be addressed by the authors.

First, in the abstract section, they claimed that ethanol lock is effective on reducing the incidence of CRBSI in hemodialysis patients with tunneled central venous catheters. However, the majority of participants in the studies included in their meta-analysis were not hemodialysis patients; there were three studies in the meta-analysis involving hematology patients receiving chemotherapy via central venous catheters and only one study involving hemodialysis patients. Therefore, the conclusion in the abstract needs to be rephrased.
Second, the meta-analysis reported thrombosis as an adverse effect of ethanol lock. However, other adverse effects, such as nausea, dizziness, flushing, and altered taste, were also reported in some of the included studies, yet they were not considered in the meta-analysis itself.

\section{Compliance with ethical standards}

Conflict of interest The authors declare that they have no conflict of interest.

\section{Reference}

1. Zhao T, Liu H, Han J (2018) Ethanol lock is effective on reducing the incidence of tunneled catheter-related bloodstream infections in hemodialysis patients: a systematic review and meta-analysis. Int Urol Nephrol. https://doi.org/10.1007/s11255-018-1855-4

Xin-Jun Su

1919519046@qq.com

Xing-Huan Wang

wangxinghuan@whu.edu.cn

1 Department of Urology, Zhongnan Hospital, Wuhan

University, 169 Donghu Road, Wuchang District,

Wuhan 430071, Hubei, China 